\title{
A Rare Cause of Acute Abdomen Due to Hemorrhage of Renal Mass: Wunderlich Syndrome
}

\author{
Umut Özdamarlar1, Ercan Ayaz², Zafer Küçükodacli, Emin Değer', Mustafa Kemal Demir' \\ 'Department of Radiology, Medical Park Göztepe Hospital, İstanbul, Turkey \\ 2Department of Radiology, Istanbul Medeniyet University School of Medicine, İstanbul, Turkey \\ ${ }^{3}$ Department of Pathology, Gülhane Military Medical Academy Haydarpaşa Training Hospital, İstanbul, Turkey
}

Cite this article as: Özdamarlar U, Ayaz E, Küçükodacı Z, Değer E, Demir MK. A Rare Cause of Acute Abdomen Due to Hemorrhage of Renal Mass: Wunderlich Syndrome. J Emerg Med Case Rep 2017; 8: 55-8.

\begin{abstract}
Introduction: Wunderlich syndrome is an emergency medical condition that refers to spontaneous nontraumatic bleeding confined to the perinephric space. Renal angiomyolipomas are the most frequent etiology. Herein, we present a case of Wunderlich syndrome whose vital signs abruptly worsened and went into hypovolemic shock and required an urgent surgical approach.

Case Report: A 55 year old woman applied to our emergency room with flank pain for 4 days, which worsened severely that morning. In her physical examination, her abdomen was tight and tender and she felt pain with palpation on the left flank. Her abdomen sonography revealed a mass in the upper pole of her left kidney consistent with angiomyolipoma. Also there was subcapsular fluid collection. Intravenous (IV) contrast enhanced computerized tomography (CT) was done immediately, which showed a $9.5 \mathrm{~cm}$ diameter mass with arterial contrast extravasation into the mass and subcapsular area. There was a large perinephric hematoma. After 2 hours in the emergency room the patient became haemodynamically unstable and entered the operation room with hypovolemic shock. Radical nephrectomy was performed.

Conclusion: Wunderlich syndrome is a rare cause of acute abdomen. Therefore, urgent and specific management to diagnose and treat it as soon as possible is needed in cooperation with emergency doctors, radiologists, and urologists.
\end{abstract}

Keywords: Acute abdomen, wunderlich syndrome, angiomyolipoma, perinephric hemorrhage

Received: 29.05.2016 Accepted: 18.10.2016 Available Online Date: 30.11.2016

\section{Introduction}

Renal angiomyolipomas are rare benign lesions of the kidney with an incidence between $0.1 \%$ and $0.22 \%$ (1). They are composed of abnormal vasculature, smooth muscle, and adipose tissue. They are more likely to be symptomatic if they are larger than $4 \mathrm{~cm}$, and present a history of flank pain, a palpable mass, and rarely haematuria. Also, they are most frequent etiology of Wunderlich syndrome, which is an emergency medical condition that refers to spontaneous nontraumatic bleeding confined to the perinephric space (2). Although benign, their rich neovascularization has a tendency for spontaneous rupture with consequent hemorrhage, which in some cases can be life threatening. Significant perinephric hemorrhage may occur in approximately $10 \%$ of patients with angiomyolipoma (3). This complication is related to the size of the tumor, the grade of the angiogenic component of the tumor, and the presence of tuberous. Herein, we present a catastrophic case of Wunderlich syndrome whose vital signs abruptly worsened, went into hypovolemic shock and required an urgent surgical approach.

\section{Case Report}

A 55 year old woman applied to our emergency room with flank pain for 4 days, which worsened severely that morning. She had a history of cholecystectomy and lomber disc hernia. On examination, she appeared pale but her vital signs

This study was presented at the $37^{\text {th }}$ Turkish National Congress of Radiology, 1-5 November 2016, Antalya, Turkey

Address for Correspondence:

Ercan Ayaz, Department of Radiology, Istanbul Medeniyet University School of Medicine, Istanbul, Turkey

E-mail: ercan_ayaz@yahoo.com

oCopyright 2017 by Emergency Physicians Association of Turkey - Available online at www.jemcr.org 

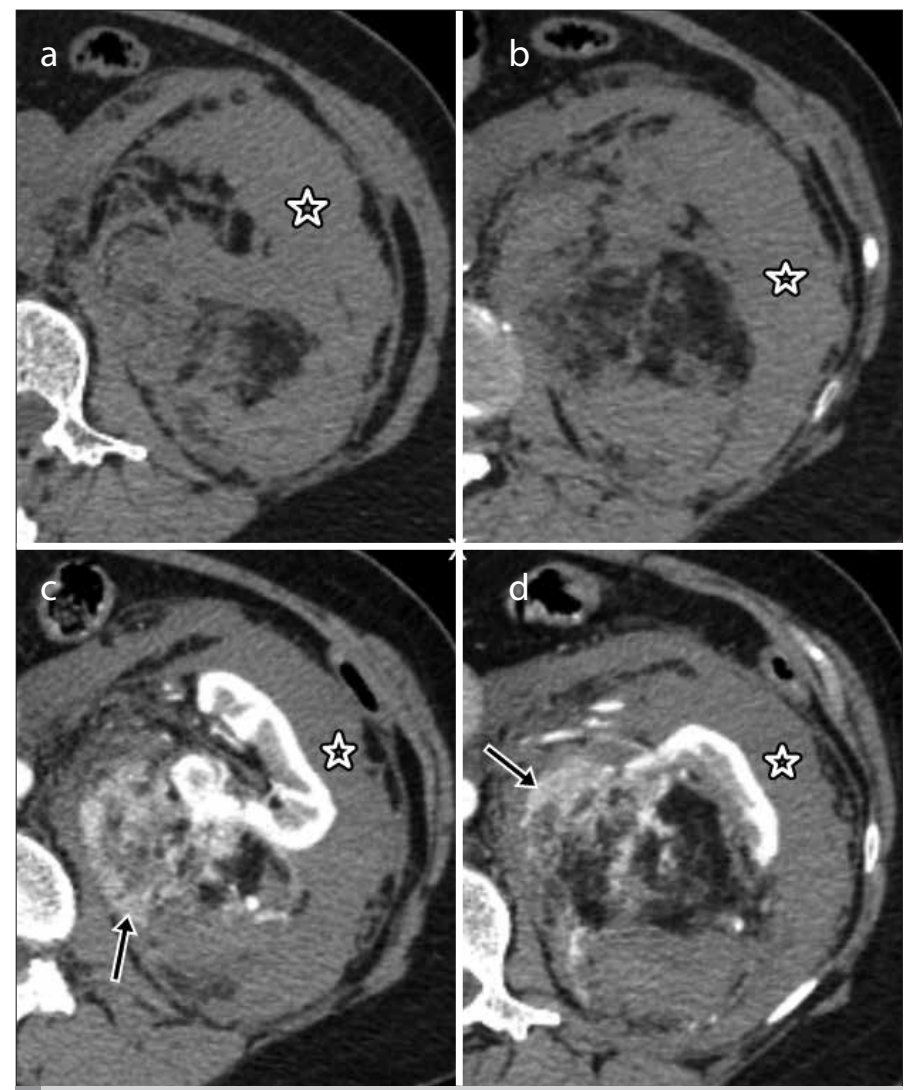

FIGURE 1. a-d. Axial noncontrast (a, b) and IV contrast enhanced (c, d) CT images show a $9.5 \mathrm{~cm}$ diameter mass in the kidney containing fat densities and contrast extravasation (arrows) into the mass and subcapsular area. There is a large perinephric hematoma (star) that displaces the left kidney anteromedially. Note the normal parenchymal contrast enhancement

were within normal range: pulse 80 , blood pressure 120/80 and oxygen saturations $97 \%$. In her physical examination her abdomen was tight and tender and she felt pain with palpation on the left flank. Blood investigations revealed a leukocytosis of (25.8 K/uL) and normocytic anemia $(11.5 \mathrm{~g} / \mathrm{dL})$. Her bleeding parameters were: A ivated partial thromboplastin time APTT $18.4 \mathrm{sn}$ and INR 1.23. Her abdomen sonography revealed a 9 $\mathrm{cm}$ diameter heterogeneous, hardly demarcated mass in the upper pole of her left kidney which includes fat echogenicity, consistent with angiomyolipoma. Also there was a $4 \mathrm{~cm}$ width subcapsuler fluid collection that was interpreted as a retroperitoneal hematoma.

Intravenous (IV) contrast enhanced computerized tomography (CT) of the abdomen was done immediately, which showed a $9.5 \mathrm{~cm}$ diameter mass in the upper pole of the left kidney containing fat densities and IV contrast extravasation into the mass and subcapsular area. There was a large perinephric hematoma that displaced the left kidney medially. The kidney's parenchymal contrast enhancement was normal (Figure 1, 2).

After 2 hours in the emergency room, the patient gradually became lethargic and pale. Her vitals worsened with a pulse of $130 \mathrm{bpm}$,

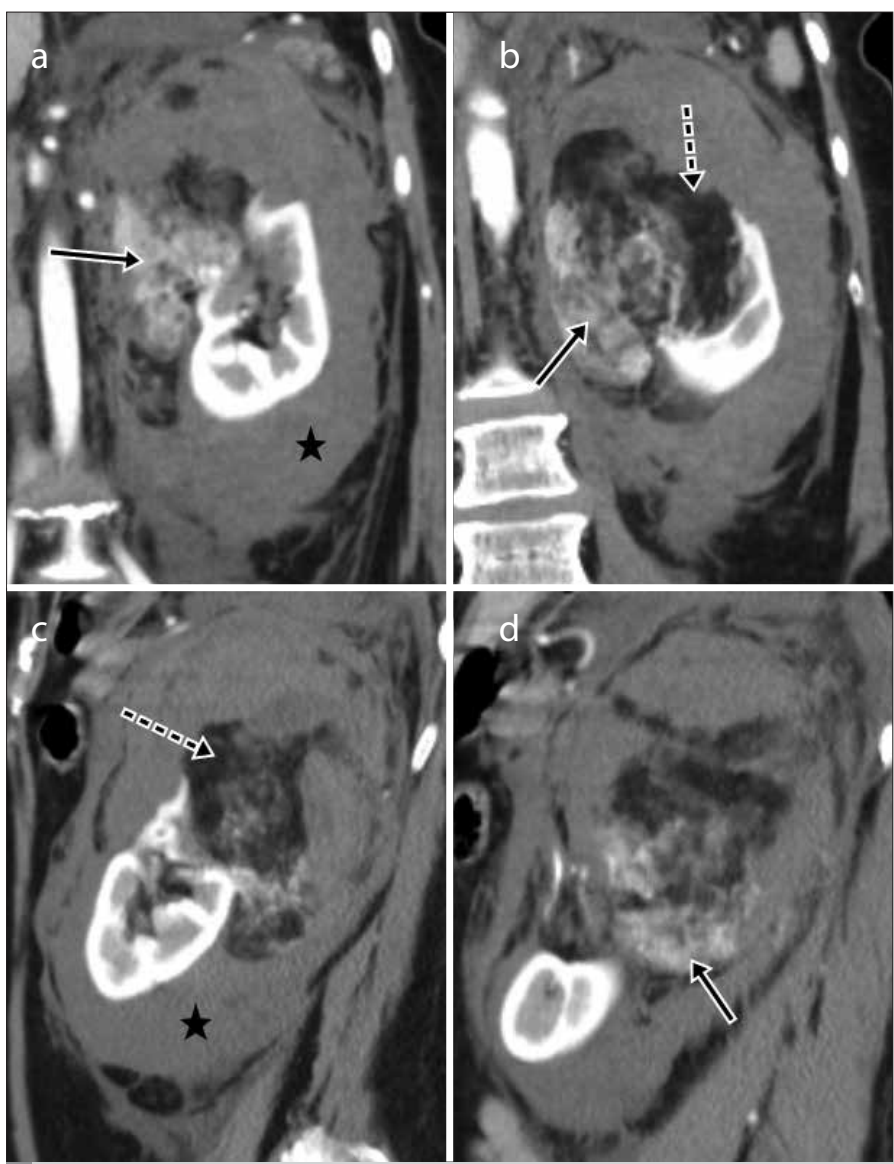

FIGURE 2. a-d. Coronal (a, b) and sagittal (c, d) reformatted CT images of the mass reveal contrast extravasation (arrows), fat containing mass (dashed arrow), and large perinephric haematoma (star)

blood pressure of $85 / 55$, and oxygen saturation of $95 \%$. The patient became haemodynamically unstable and a laparotomy was crucial.

The patient entered the operation room with hypovolemic shock. Her abdomen was opened with a left paramedian incision and the left colon was freed from its attachments and moved medially. A hematoma and giant mass with hemorrhage were seen in the left retroperitoneum. The mass was perforated before surgery. The ureter was cut, the left renal artery and vein were tied and radical nephrectomy was performed without opening Gerota's fascia. With stable vital findings the patient was interned to the intensive care unit. After a week the patient was discharged with full recovery. The 3 months follow up sonography showed no residual lesion. Patient was informed about his results and written consent for publishing this case report was obtained.

At the histopathologic examination, the resected kidney revealed a large haematoma within the perinephric space and a ruptured tumor at the lower pole (Figure 3a). Microscopically, the tumor was shown to contain proportions of mature adipocyte clusters divided with thin fibrous bands, myoblastic cells and small blood vessels composed of proliferated endothelial cells, which confirmed the neoplasm to be a hemorrhagic benign angiomyolipoma (Figure 3b). 

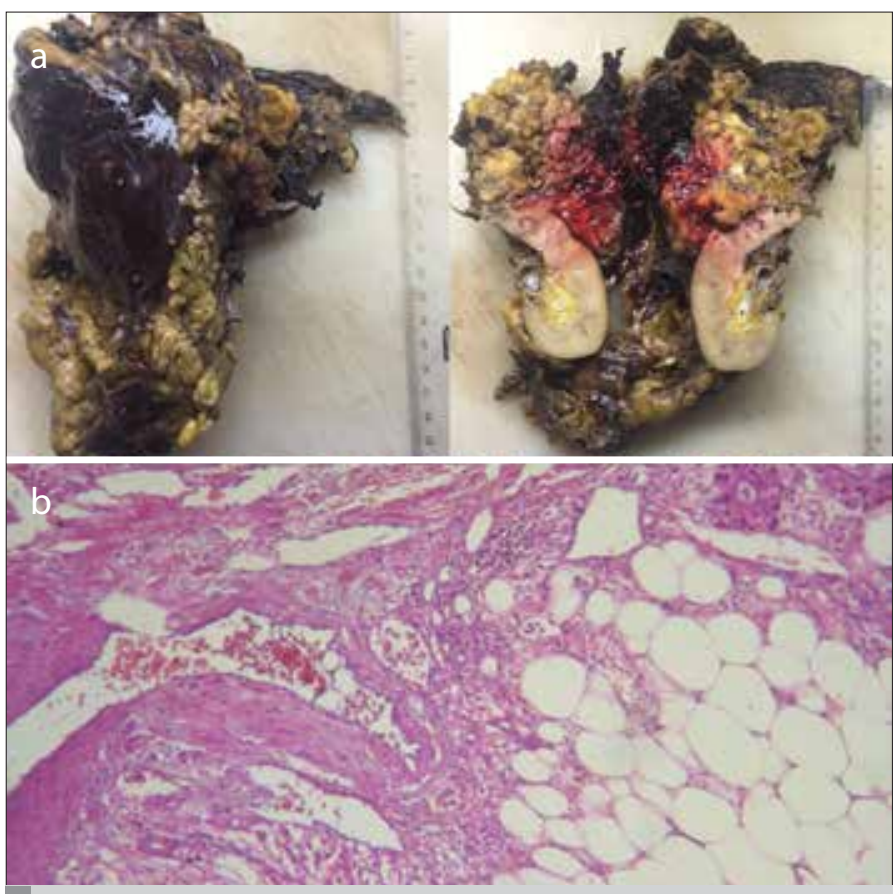

FIGURE 3. a, b. (a) Macroscopic view of the hemorrhagic tumor and left kidney and (b) histologically the tumor consists of adipose tissue, vessels, and immature leiomyoblasts

\section{Discussion}

Spontaneous bleeding confined to the subcapsular and perinephric area was initially described by Wunderlich in 1856 (4). The classic triad of symptoms is as follows: acute flank pain, palpable mass, and general deterioration of vital signs (5). There are a wide range of etiologies such as renal tumors, vascular lesions such as polyarteritis nodosa, renal infections, cysts, hematological conditions and hypertension, but the most common cause among them in most series, similarly to our case, is angiomyolipoma (6).

Angiomyolipoma is made of fat, smooth muscle and thick-walled blood vessels. Two subtypes of angiomyolipoma have been described in the literature: isolated angiomyolipoma and angiomyolipoma associated with tuberous sclerosis. Isolated angiomyolipoma is often solitary and accounts for $80 \%$ of angiomyolipomas. Angiomyolipomas are generally asymptomatic and found incidentally during imaging for another reason. Symptomatic patients may present with a combination of flank pain (53\%), a palpable tender mass (47\%) and gross haematuria (23\%), which is known as Lenk's triad (7). Wunderlich syndrome is a fatal complication of angiomyolipoma which can occur in up to $50 \%$ of patients with tumors larger than $40 \mathrm{~mm}$, whereas $33 \%$ of the patients with bleeding angiomyolipoma can develop hypovolemic shock (8). Angiomyolipoma includes abnormal elastin-poor vascular structures that can easily lead to aneurysm formation and rupture (9).

The rarity and nonspecific presentation of Wunderlich syndrome lead to the challenge of diagnosis. Ultrasound is generally the first choice for perinephric hemorrhage because it is quick and inexpensive. However CT scans are often needed to confirm sonogram findings and to rule out an underlying mass. CT performed at the time of hemorrhage is only moderately successful in identifying the renal neoplasm causing hemorrhage, however, it has been found to identify all cases of Wunderlich syndrome due to angiomyolipoma because of its macroscopic fat content (6). Other renal tumors, such as renal cell carcinoma, lipoma, liposarcoma, oncocytoma and Wilm's tumor, may also show fat content, but it has been thought that a renal cortical mass that shows a predominantly fat attenuation of less than $-20 \mathrm{HU}$ can be confidently diagnosed as an angiomyolipoma.

The treatment options of spontaneous perinephric hemorrhage due to angiomyolipoma depend on the general status of the patient, the size of the tumor and hemorrhage. Most patients present stable vital signs and can be managed conservatively and deferred partial nephrectomy can be performed. Embolisation is very useful in the acute setting of hemorrhage due to angiomyolipoma rupture (10). In our case, the patient was presented as an emergency with an acute abdomen, and rapid onset of hypovolemic shock. This unusual presentation necessitated emergency life-saving open surgery and radical nephrectomy.

\section{Conclusion}

Wunderlich syndrome, which is a rare cause of acute abdomen, needs urgent and specific management to diagnose and treat as soon as possible in cooperation with emergency doctors, radiologists, and urologists.

We hope that our case report helps to raise clinicians' awareness of Wunderlich syndrome in the onset of acute abdomen and rapid deterioration of a patient's status. Early use of IV contrast enhanced $\mathrm{CT}$ in appropriate circumstances and a multidisciplinary team may allow proper treatment.

Informed Consent: Written informed consent was obtained from the patient who participated in this study.

Peer-review: Externally peer-reviewed.

Author contributions: Concept - Z.K., M.K.D.; Design - E.A.; Supervision - M.K.D.; Resource - Z.K., U.Ö.; Materials - Z.K., U.Ö.; Data Collection and/or Processing - E.A., U.Ö.; Analysis and/or Interpretation - U.Ö., M.K.D.; Literature Search - E.A., U.Ö.; Writing - E.A.; Critical Reviews - M.K.D.

Conflict of Interest: No conflict of interest was declared by the authors.

Financial Disclosure: The authors declared that this study has received no financial support.

\section{References}

1. Oesterling JE, Fishman EK, Goldman SM, Marshall FF. The management of renal angiomyolipoma. J Urol 1986; 135: 1121-4.

2. Fujii Y, Ajima J, Oka K, Tosaka A, Takehara Y. Benign renal tumors detected among healthy adults by abdominal ultrasonography. Eur Urol 1995; 27: 124-7.

3. Moratalla MB. Wunderlich's syndrome due to spontaneous rupture of large bilateral angiomyolipomas. Emerg Med J 2009; 26: 72. [CrossRef]

4. Wunderlich CRA. Handbuch der Pathologie und Therapie. 2 nd ed. Stuttgart: Ebner\& Subert 1856 
5. Albi G, del Campo L, Taggaro D. Wünderlich's Syndrome: causes, diagnosis and radiological management. Clin Radiol 2002; 57: 840-5. [CrossRef]

6. Zhang JQ, Feilding JR, Zou KH. Etiology of spontaneous perirenal hemorrhage: a meta-analysis. J Urol 2002; 167: 1593-6. [CrossRef]

7. Simmons JL, Hussain SA, Riley P, Wallace DM. Management of renal angiomyolipoma in patients with tuberous sclerosis complex. Oncol Rep 2003; 10: 237-41. [CrossRef]
8. Chan CK, Yu S, Yip S, Lee P. The efficacy, safety and durability of selective renal arterial embolization in treating symptomatic and asymptomatic renal angiomyolipoma. Urology 2011; 77: 642-8. [CrossRef]

9. Eble JN. Angiomyolipoma of kidney. Semin Diagn Pathol 1998; 15: $21-$ 40.

10. Nelson CP, Sanda MG. Contemporary diagnosis and management of renal angiomyolipoma. J Urol 2002; 168: 1315 -25.[CrossRef] 